\title{
Cell segmentation and pipette identification for automated patch clamp recording
}

\author{
Runhuai Yang ${ }^{1,2}$, Chi Ho Tam ${ }^{1}$, Ka Lun Cheung ${ }^{1}$, Kai Chun Wong ${ }^{1}$, Ning Xi ${ }^{3}$, Jie Yang ${ }^{2}$ and King Wai Chiu Lai ${ }^{1 *}$
}

\begin{abstract}
A visual-based approach for identifying living cells and performing the automated patch clamp recording was reported. Based on the image processing and blob detection algorithm, the vision-based method was developed for the detection and identification of biological cells and micropipette. The method was implemented in a micromanipulation system that enabled the identification of the boundary and the center of the target cell and separation from its neighboring cells. The method successfully identified a batch of neuroblastoma cells with the highest yield of 90\%. The results demonstrated that the visual-based approach can be integrated to the micromanipulation system to automatically manipulate the patch pipette tip to the center of the target cell, and as a result, the whole-cell recording can be performed precisely and effectively.
\end{abstract}

\section{Background}

Patch clamp technique is one of the promising intracellular bio-electrical signal recording methods for measuring the electric current flowing through ion channels in a cell membrane [1]. It is well understood that the ion channels played important roles in regulating the flow of ions across the cell membrane, which are involved critically in various physiological processes [2]. Therefore, patch clamp recording was widely used for studying the electrophysiological properties of cells [3] and applied for investigating the effect of different drugs on the cell [4]. In addition, patch clamp was specially suitable for high-resolution current measurements under low noise by using the patch clamp pipette and applying a known voltage across the membrane [5]. Therefore, the adoption of the patch clamp technique has led to significant advances in the life sciences and biomedical studies, including the characterization of the properties of ion channels in various types of cells and the pharmacological treatment of diseases implicated with ion channels such as diabetes and heart disease [6] and Parkinson's disease [7].

However, the current patch clamp method required a skilled technician to manually manipulate the pipette, apply the appropriate pressure, and observe the electrical

\footnotetext{
* Correspondence: kinglai@cityu.edu.hk

'Department of Mechanical and Biomedical Engineering, City University of

Hong Kong, Tat Chee Ave, Kowloon, Hong Kong

Full list of author information is available at the end of the article
}

resistance [8]. This complicated operation normally requires several months or even 1 year of training before the technician is able to reliably record from the cells [9] because of the difficulty in obtaining successful recording. This also significantly increases the cost of the whole patch clamp recording process. Due to these challenging issues, several groups started incorporating automated systems to current patch clamping so as to reduce the operation time, complexity, and cost. The automated patch clamp technology was previously reported for the integrative analysis of molecular, anatomical, and electrophysiological properties of a single cell [10]. The planar patch clamp is another kind of patch clamp systems that provides the automatic recording of cells [11]. More recently, the lateral patch clamp device was demonstrated to provide the automatic whole-cell recording [12]. The micro glass pipette has also been used for automated patch clamp $[9,13]$. Compared to the methods of planar and lateral patch clamp, the micro glass pipette provided better seal formation [14].

The current automated patch clamp system normally applies a negative pressure to catch the suspended cell in a buffer medium. This method causes some problems, for example, the tip of the pipette is blocked by the contaminant in the buffer. Moreover, the automated patch clamp system is currently difficult to fully determine the condition of the cell before catching and selecting the cell for patch clamp recording. Additionally, most of the 
current automated patch clamps were performed on the suspended cells, and the cells were fixed in the fluid medium by either mechanical method, e.g., cell scrapers or chemical method using trypsin [11], which would change the property of the cells $[15,16]$. An automated patch clamp is still difficult to be performed on those adherent cells that are grown directly on the substrate. Therefore, it is necessary to develop an automated micromanipulation system for manipulating the glass pipette to perform patch clamp recording on adherent cells.

To overcome the above limitations, we aim at designing a visual-based approach together with an image processing method for the automated patch clamp system. The system will be demonstrated to identify cells and pipettes automatically and perform patch clamp recording non-blindly on adherent cells. The typical process of the patch clamp recording on the adherent cells is shown in Figure 1. In the traditional manual operation, the target cell should be chosen manually. By observing and identifying the cells and glass pipette under the microscope (Figure 1a), the technician can manipulate the glass pipette to the cells as shown in Figure 1b. Here, the visualbased approach is designed to perform this task by the system automatically.

Several methods based on computer vision were reported which have potential to be used for automated patch clamp. The method based on template matching was used to successfully identify sperm cells [17] and other suspended cells [18]. Its concept is to find the sub-region in the input image that best matches the pre-defined template. However, the template of adherent cells is hardly described because the pattern of adherent cells is quite different with each other. It is difficult to use the template-matching method to identify adherent cells with high yield. Feature matching is another widely used matching algorithm to identify cells by matching the nuclei of the cells because the nuclei of same type of cells have similar features [19]. However, the limitation of this method is that the height of the nuclei of the cells should be much higher than other regions of the cells. The yield of identification is strongly affected by the condition of the cells. In this paper, the blob detection will be used as the main method to detect cells and pipettes for patch clamp. Blobs are binary objects or points which are in the same state [20]. Blobs can be classified as different objects based on color, shape, area, perimeter, etc. [21]. This method can thus be applied to identify the target cell based on its own shape and feature and separate the cell from other neighboring cells in the same image. Suspended cells such as red blood cells and white blood cells have been successfully detected and distinguished by using the blob detection method [22]. For detecting adherent cells, the situation is more complicated than the suspended cells because the pattern of adherent cells is varied. Moreover, the adherent cells after several days of culture are grown quite close to each other. Therefore, it is necessary to separate the neighboring cells during the identification process so as to avoid the wrong identification. For pipette detection, some methods were used to identify the pipettes for cell injection [23] or micromanipulation [24]. However, these methods cannot be directly used for the identification of the patch pipette in the patch clamp recording. It is because the pipette and cells are located closely.

To overcome these challenges, a method for detection and identification of a single cell and pipette for automated patch clamp is developed in this paper. More

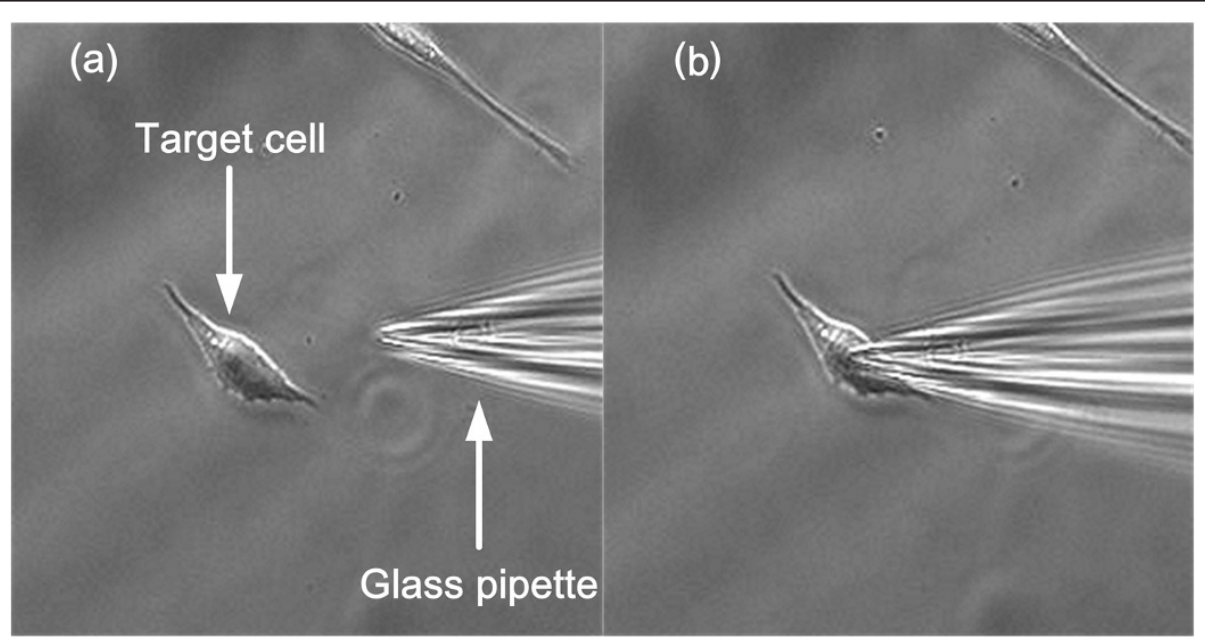

Figure 1 Optical images showing two states of the patch clamp recording process. (a) The initial state before the patch clamp recording, which indicates the initial position of the target cell and patch pipette and (b) the pipette is successfully moved to the center of the cell and patched on the cell. 
importantly, we report the novel use of the visual-based approach for automatically identifying the target adhered cells and pipettes. Based on the position and feature of the cells and pipette, the condition of the cell can be revealed more clearly, and the cell can be selected for automated patch clamp recording. The method is further combined with the automatic control techniques for performing automated patch clamp on adherent cells, which is different with the current non-visual-based automated patch clamp system.

\section{Methods}

\section{Cell preparation}

Neuroblastoma cells were employed as the sample cells for the identification and patch clamp recording. The neuroblastoma cells were cultured in Dulbecco's modified eagle medium containing nutrient mixture F-12 (Gibco, Thermo Fisher Scientific Inc., Waltham, MA, USA) with $10 \%$ fetal bovine serum (Gibco, USA) and $1 \%$ penicillin-streptomycin-neomycin antibiotic mixture (Gibco, USA) and maintained at $37^{\circ} \mathrm{C}$ in $5 \% \mathrm{CO}_{2}$ humidified air. Stock cultures were passaged at 1:6 weekly and fed twice weekly. The cells were subcultured with the cell density of 100,000 in a culture flask. During the process of subculture, the cells were detached from the culture flask by the treatment of $1 \mathrm{ml}$ trypsin-EDTA (Gibco, USA). Around 5,000 cells were then counted and seeded onto the cover-slip coated with Poly-D-lysine hydrobromide (Sigma-Aldrich, St. Louis, MO, USA) and cultured for 6 days before patch clamp recording. These cells were induced to differentiate by the treatment with $10 \mu \mathrm{M}$ retinoic acid. All the experiments were conducted at $37^{\circ} \mathrm{C}$.

\section{Experimental setup and control strategy}

The illustration of the experimental setup for implementing the patch clamp recording based on the visualbased identification is shown in Figure 2. The cells and pipette were first observed under an inverted microscope (Olympus, Shinjuku-ku, Japan). The microscope was equipped with four different objectives with the magnification of $\times 4, \times 10, \times 20$, and $\times 40$. The live video and digital real-time image were then captured using a CCD camera. The resolution of the image was $1,280 \times$ 768 pixels. Based on the image processing and identification method as described in Figure 2, the coordinates of the cell and the pipette can be obtained from the image, and the movement of the manipulator was then calculated.

To achieve the automated patch clamp, the patch pipette was mounted to a computer-controllable micromanipulator (Sutter MP-285; Sutter Instrument Company, Novato, CA, USA) connected to a controller (Sutter MPC200, USA). The micromanipulator was controlled to

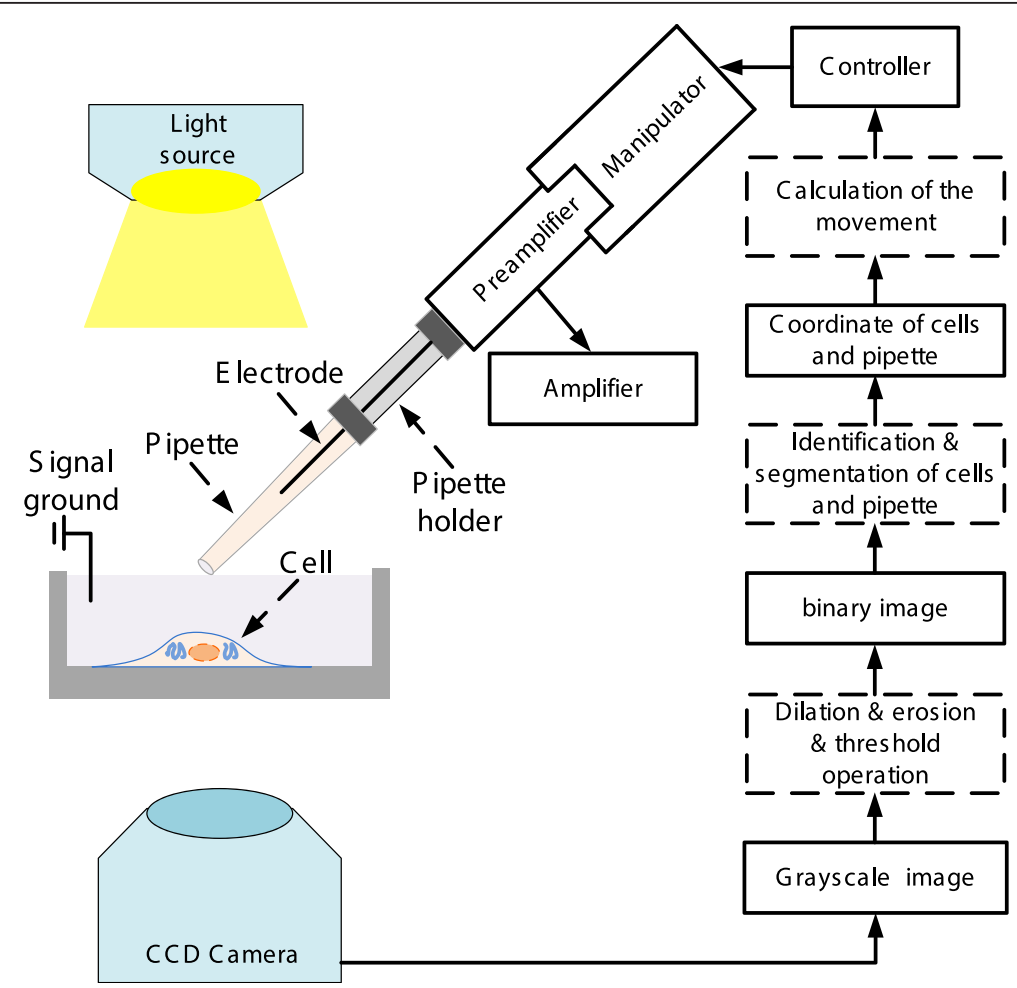

Figure 2 Experimental setup for performing the automated patch clamp based on the developed identification method. 


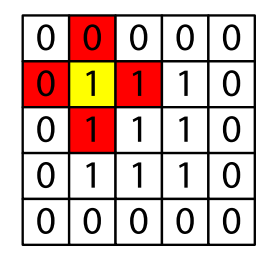

(a)

\begin{tabular}{|l|l|l|l|l|}
\hline 0 & 1 & 1 & 1 & 0 \\
\hline 1 & 1 & 1 & 1 & 1 \\
\hline 1 & 1 & 1 & 1 & 1 \\
\hline 1 & 1 & 1 & 1 & 1 \\
\hline 0 & 1 & 1 & 1 & 0 \\
\hline
\end{tabular}

(b) (c)

\begin{tabular}{|l|l|l|l|l|}
\hline 0 & 0 & 0 & 0 & 0 \\
\hline 0 & 0 & 0 & 0 & 0 \\
\hline 0 & 0 & 1 & 0 & 0 \\
\hline 0 & 0 & 0 & 0 & 0 \\
\hline 0 & 0 & 0 & 0 & 0 \\
\hline
\end{tabular}

Figure 3 Processes of dilation and erosion operations. (a) A $3 \times$ 3 cross shape structural element applied to a $5 \times 5$ binary image. Each pixel value in the element represented the intensity. (b) Output image obtained from dilation. (c) Output image obtained from erosion.

move along the $\mathrm{x}_{-}, \mathrm{y}_{-}$, or $\mathrm{z}$-axis with the range from 0 to $25,000 \mu \mathrm{m}$. The micromanipulator was then moved to the desired position based on the command received from the controller. The pipette was connected to the manipulator via a pipette holder, and the position of pipette tip was then controlled by the manipulator. The amplifier was used to perform the patch clamp recording. The signal ground of the patch clamp amplifier was connected to the extracellular buffer in the dish. The patch clamp amplifier applied a voltage pulse to the cell and the corresponding current between the electrode inside the pipette and the signal ground was then recorded. By monitoring the current, the condition of the pipette and the cell membrane was determined.

The automatic control strategy can be divided into four steps. In the first step, a digital image containing cells and pipette is captured by the CCD camera, and the system transforms the digital image to the grayscale image. In the second step, the dilation, erosion, and threshold operation are applied to the grayscale image, and the binary image is then automatically obtained. In the third step, the boundary of the cell is identified, and the center of the cell is calculated from the binary image. Besides, the tip of the pipette is also displayed and identified in the binary image. Finally, the coordinate of the center of the cell and the tip of the pipette are obtained. The movement from the tip of the pipette to the center of the cell is calculated based on the updated coordinates, and the command is sent to the controller for moving the manipulator and the pipette tip to the center of the cell. The movement of the pipette tip can be adjusted based on the updated coordinates of the cell and the pipette.

\section{Identification of cells}

To perform the detection of the cell and pipette for patch clamp, the digital image of the sample was first transformed to a grayscale image. In the grayscale image, every pixel was represented by an intensity value ranging from 0 to 255 . The image was then processed by applying two basic morphological operations, namely dilation and erosion [25]. The concept of the dilation operation was to increase the area of bright regions in the image as the erosion was to increase the area of dark regions in the image. In order to perform the appropriate morphological operation to the image, a structural element, a matrix consisting of only 0 and 1 values, was defined and used. As shown in Figure $3 \mathrm{a}$, a $3 \times 3$ cross shape structural element was applied to a $5 \times 5$ binary image, and each pixel value in the element represented the intensity. When the dilation was applied, the maximal pixel value overlapped by the structural element was found to replace the pixel value at the center of the structural element. Therefore, the resulting bright region was increased after performing the dilation as shown in Figure $3 \mathrm{~b}$. When the erosion was applied, the minimal pixel value overlapped by the structural element was found, and the pixel value at the center of the structural element was then replaced by this minimal pixel value. Therefore, the resulting bright region was decreased after performing the erosion as shown in Figure 3c.

The process flow of applying the morphological operation to the cell image is shown in Figure 4. As shown in the grayscale image of the cell in Figure 4a, the cell exhibited higher intensity in the region of the cell membrane as compared with that was displayed in other areas of the cell. In order to allow the system to detect and obtain the cell

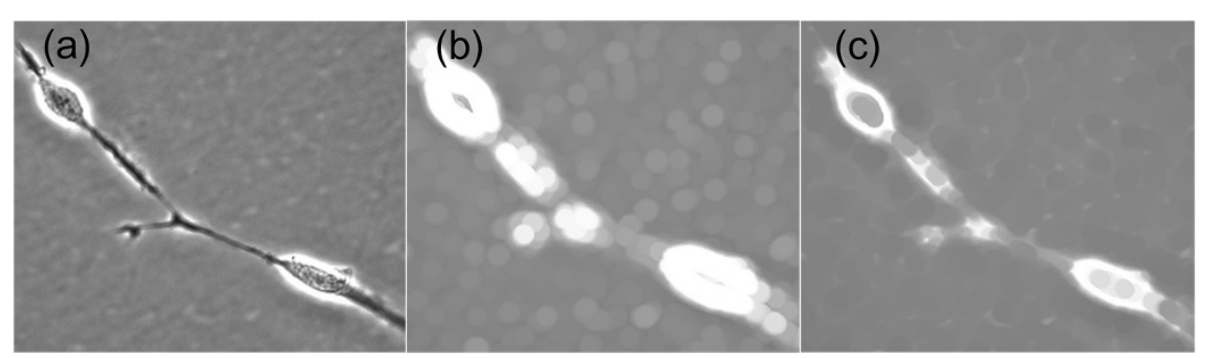

Figure 4 Images obtained before and after dilation and erosion operations. (a) Grayscale image of cells; (b) cell image obtained from dilation; and (c) cell image obtained from dilation followed by erosion. 


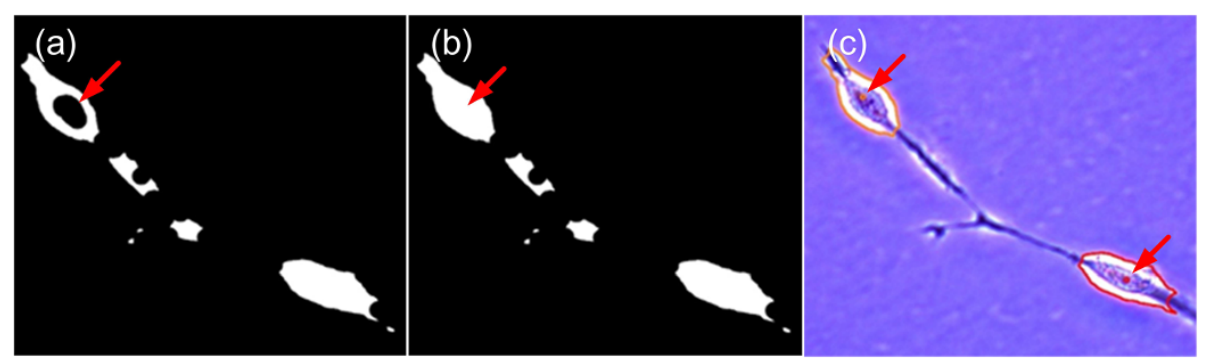

Figure 5 Image obtained by using threshold operations and centers obtained by calculation. (a) Cell image after applying threshold operations. (b) The black pixels inside the white pixels (indicated by red arrow in (a)) are filled. (c) Image showing the detected cells (the boundary is indicated by red lines) and their centers are indicated by red arrows.

outline, the dilation followed by erosion was applied to the image as shown in the output image of the cells in Figure $4 \mathrm{c}$, the cell boundary with higher intensity than the background was generated.

After performing the dilation and erosion of the cell image, the cells were further segmented from the background by applying threshold operations to the image. For a grayscale image with intensity value ranging from 0 to 255 , pixels with an intensity value that is higher than the threshold were changed to the maximum intensity value, i.e., 255 , while pixels with the intensity value equal or lower than the threshold were

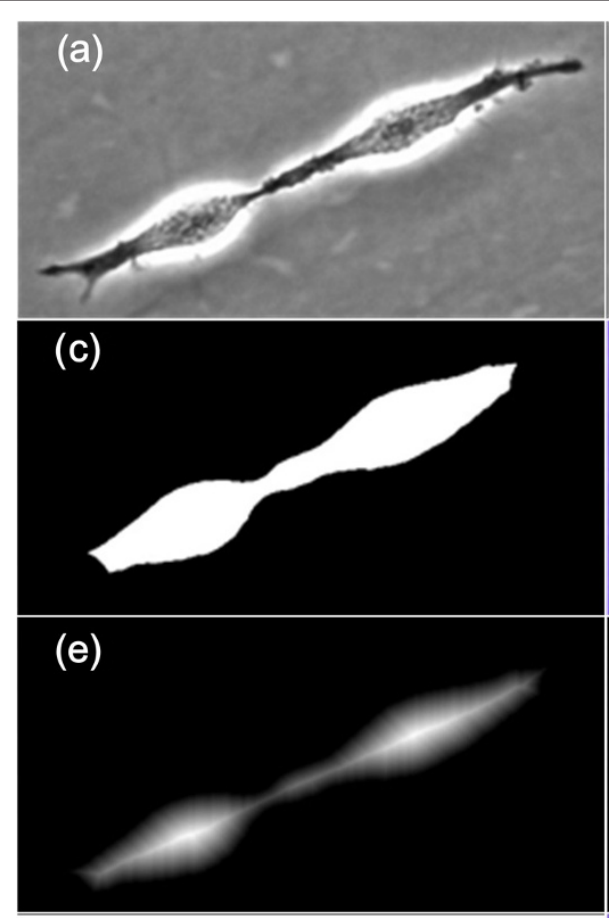

(g)

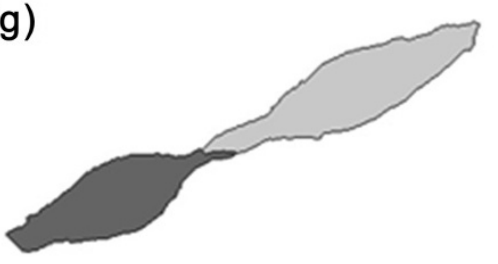

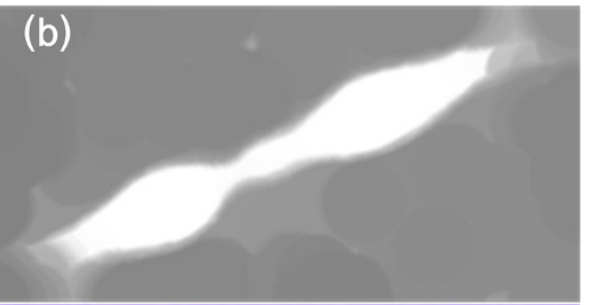
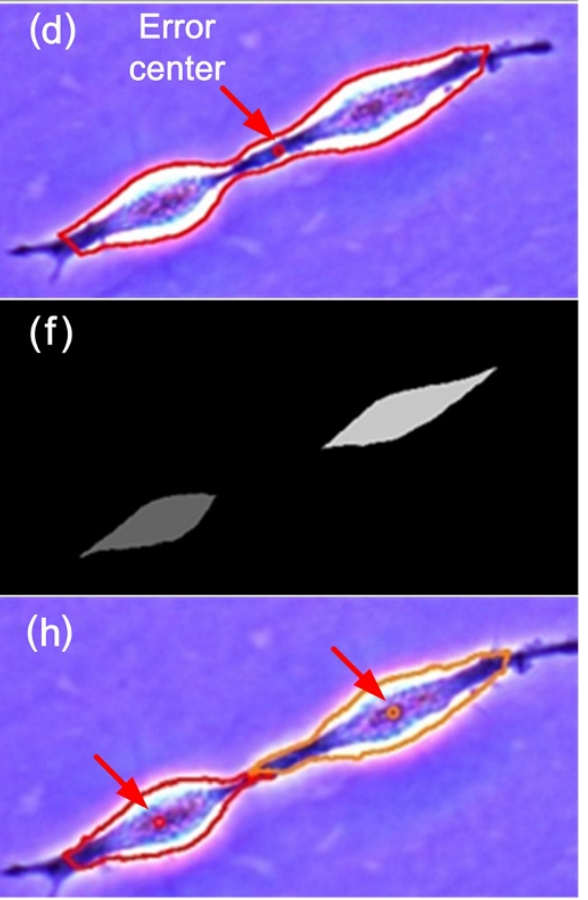

Figure 6 Processes of segmentation of neighboring cells. (a) Grayscale image of two adjacent cells which are closed to each other; (b) grayscale image, (c) binary image, and (d) the matching result obtained from morphological operation (dilation followed by erosion); (e) distance transform image; (f) markers of the cells and background in different gray level values; (g) result of applying watershed transformation using the markers; (h) corrected identification of the two cells (the boundaries are indicated by red lines and their centers are indicated by red arrows). 


\begin{tabular}{|l|l|l|l|l|l|l|l|l|l|l|l|l|l|}
\hline 0 & 0 & 0 & 0 & 0 & 0 & 0 \\
\hline 0 & 1 & 1 & 1 & 1 & 1 & 0 \\
\hline 0 & 1 & 1 & 1 & 1 & 1 & 0 & 0 & 0 & 0 & 0 & 0 & 0 \\
\hline 0 & 1 & 1 & 1 & 1 & 1 & 0 \\
\hline 0 & 1 & 1 & 1 & 1 & 1 & 0 & 2 & 2 & 2 & 1 & 0 \\
\hline 0 & 1 & 1 & 1 & 1 & 1 & 0 & 1 & 2 & 3 & 2 & 1 & 0 \\
\hline 0 & 1 & 1 & 2 & 2 & 2 & 1 & 0 \\
\hline 0 & 1 & 1 & 1 & 1 & 1 & 0 & 0 & 1 & 1 & 1 & 1 & 1 & 0 \\
\hline 0 & 0 & 0 & 0 & 0 & 0 & 0 & 0 & 0 & 0 & 0 & 0 & 0 & 0 \\
\hline
\end{tabular}

(a)

(b)

Figure 7 Distance transform. (a) Original binary image and (b) its distance transform.

changed to 0 . This operating process can be represented by the following equation:

$$
d s t(x, y)=\left\{\begin{array}{c}
\text { MaxVal if } \operatorname{src}(\mathrm{x}, \mathrm{y})>\text { threshold } \\
0 \text { if } \operatorname{src}(\mathrm{x}, \mathrm{y}) \leq \text { threshold }
\end{array}\right.
$$

where $d s t(\mathrm{x}, \mathrm{y})$ represents the intensity value of each pixel in the output image, $\operatorname{src}(\mathrm{x}, \mathrm{y})$ represents the intensity value of each pixel in the input image, and threshold is the threshold value.

After the threshold operation, a binary image was obtained in which the white areas indicated the cell features and the black areas indicated the background as shown in Figure 5a. In the next step, the black areas inside the white area were filled with white color as shown in Figure $5 \mathrm{~b}$. The cells were then identified by detecting the white areas.

After identifying the cells, the center of the cell was calculated by using the following equations:

$$
C_{x}=\frac{\text { Sum of } x \text {-coordinate within the blob }}{\text { Number of pixels within the blob }}
$$

$$
C_{y}=\frac{\text { Sum of y-coordinate within the blob }}{\text { Number of pixels within the blob }}
$$

where $C_{x}$ is the x-coordinate of the center of the cell, $C_{y}$ is the y-coordinate of the center of the cell. Finally, the membrane and the center of the cells were identified as shown in Figure 5c.

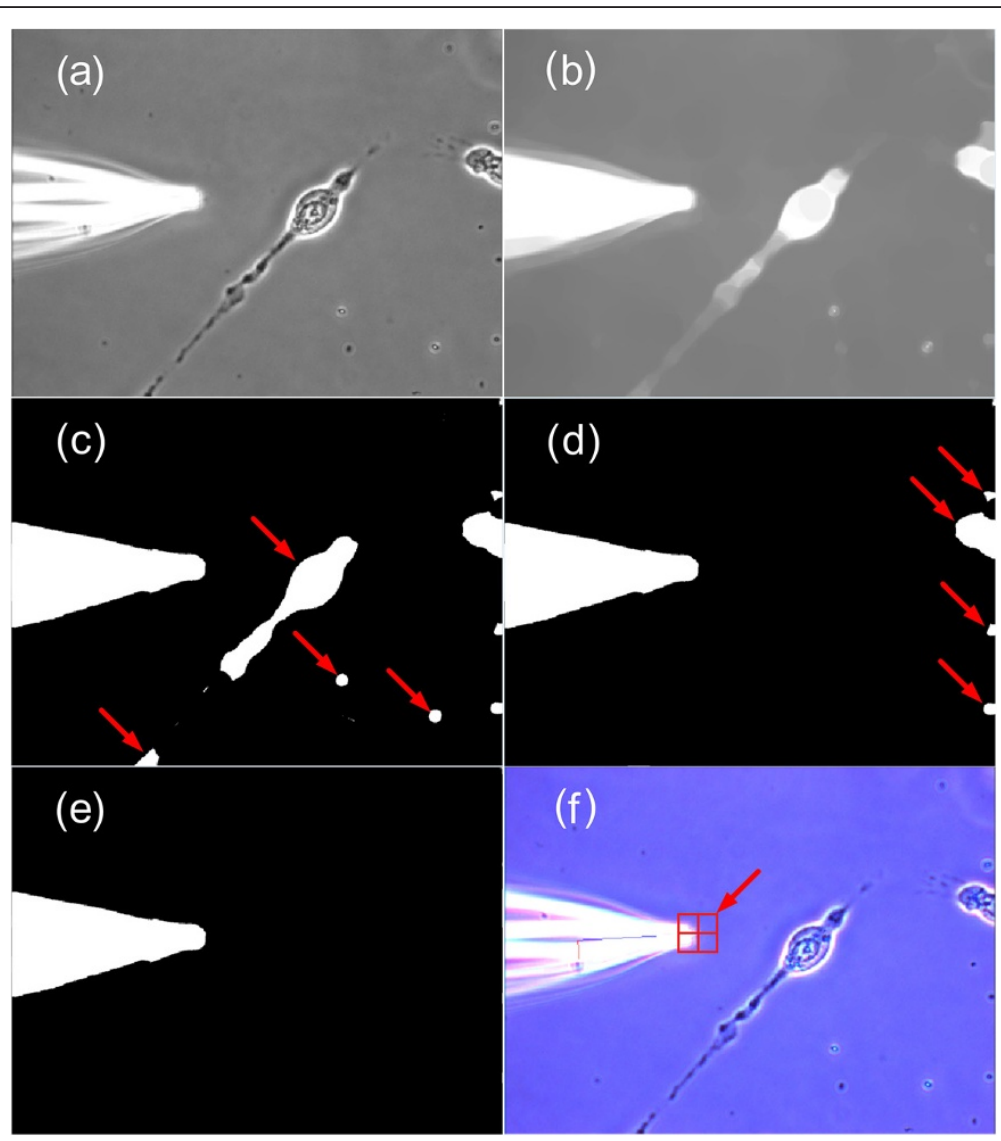

Figure 8 Processes of identification of pipette. (a) Grayscale image of a pipette and cell; (b) image obtained after dilation followed by erosion; (c) binary image of the pipette; (d) the blobs which are not connected to the boundary (indicated by red arrows in (c)) are filled with black pixels; (e) only the blob which has the longest distance in the $\mathbf{x}$-direction is left; (f) the tip of the pipette is successfully identified. 


\section{Segmentation of neighboring cells by using distance transform and watershed transformation}

Further methods were used to segment the cell from neighboring cells when the cells were too close to each other. As shown in the grayscale image of two adjacent cells (Figure 6a), the two cells were considered as one blob after the morphological operation (dilation followed by erosion (Figure 6b,c), and therefore, the center of the cells was found at the wrong position as shown in Figure 6d.

Two algorithms, distance transform and watershed transformation, were used to further process the image obtained from the morphological operation and segment the connected cells accordingly. The distance transform was first applied to the binary image, and the basic model for applying the distance transform is shown in Figure 7. Consider the binary image as shown in Figure $7 \mathrm{a}$, the foreground was extracted, and distance transform was applied from the boundary of the foreground region in the binary image. Each pixel in the foreground regions in the binary image was replaced by a pixel with gray value that was determined from the computed distance between that pixel to the closest boundary in its foreground region [26] (Figure 7b). Based on this method, the binary image of the cells after distance transform was obtained as shown in Figure 6e.

Threshold operations were then applied to the distance transform image. After the distance transform image was obtained (shown in Figure 6e), another binary image can be generated by the threshold operations. The pixels having intensity value equal or lower than the threshold were changed to 0 . Therefore, the area of the cells in the binary image generated by the threshold operations (shown in Figure 6f) was smaller than the area of the cells in the distance transform image (shown in Figure 6e). These smaller foreground regions represent the markers of the cells as shown in Figure 6f. Moreover, after the binary image was generated by the threshold operations, markers can be generated from the different blobs in the binary image. After obtaining the markers of the cells and the background as well as the gradient image of the cells as shown in Figure 6f, the watershed transformation [27] was used, and the markers were colored with different gray levels, i.e. white color for background, lighter gray color for the upper cell, and darker gray color for the bottom cell. In the watershed transformation, the lighter gray maker spreads over all pixels of the upper cell while the darker gray marker spreads over all pixels of the bottom cell. When different markers merged together, they can be recognized from different sources, and thus watershed lines were drawn to separate them as shown in Figure $6 \mathrm{~g}$. The cells were finally segmented and identified as shown in Figure $6 \mathrm{~h}$.

\section{Identification of pipettes}

The method for detection of pipette was developed when the pipette and cells appeared in the same image. The pipette was first calibrated to achieve two conditions
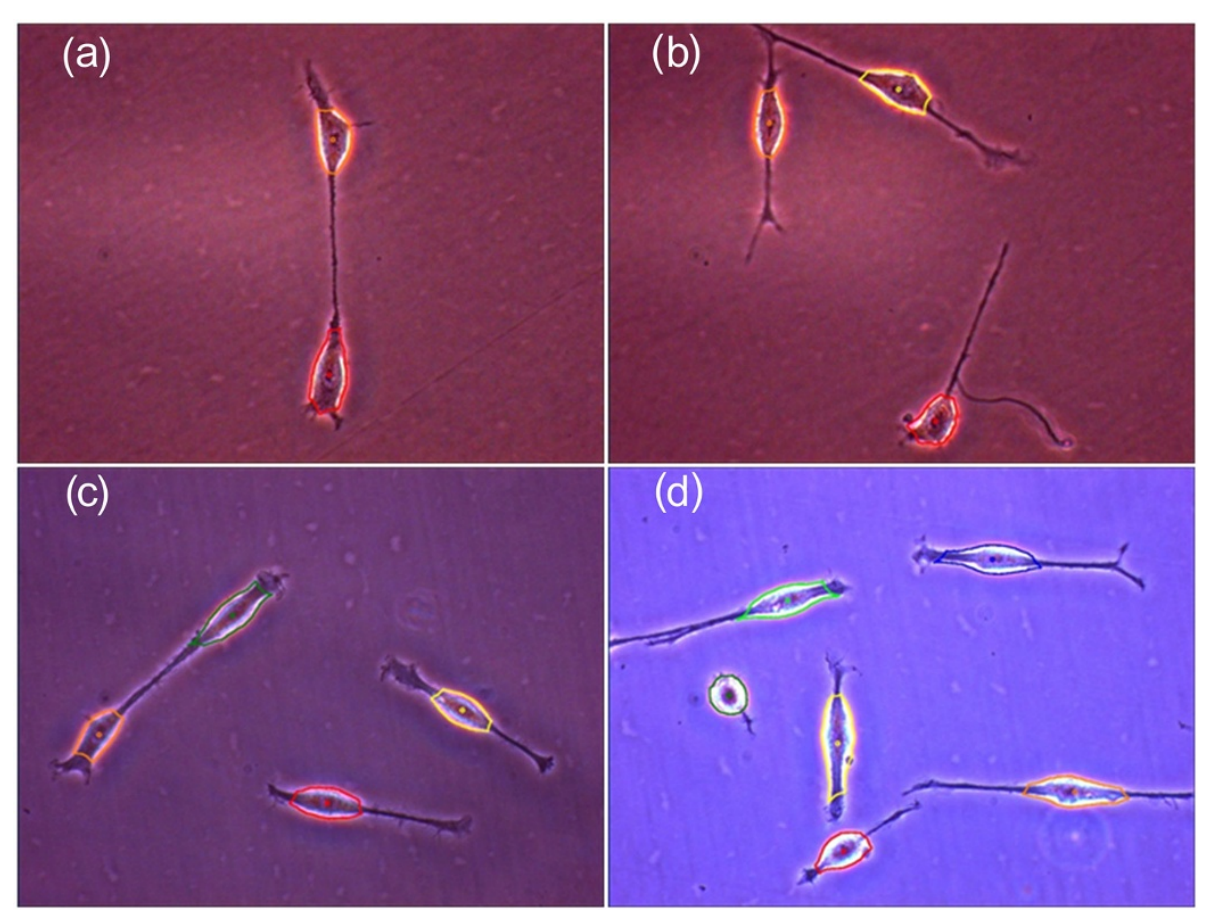

Figure 9 Different shapes and numbers of cells were successfully identified with the marked cell centers and boundaries (a-d). The boundary of each cell is indicated by the line in different colors. 

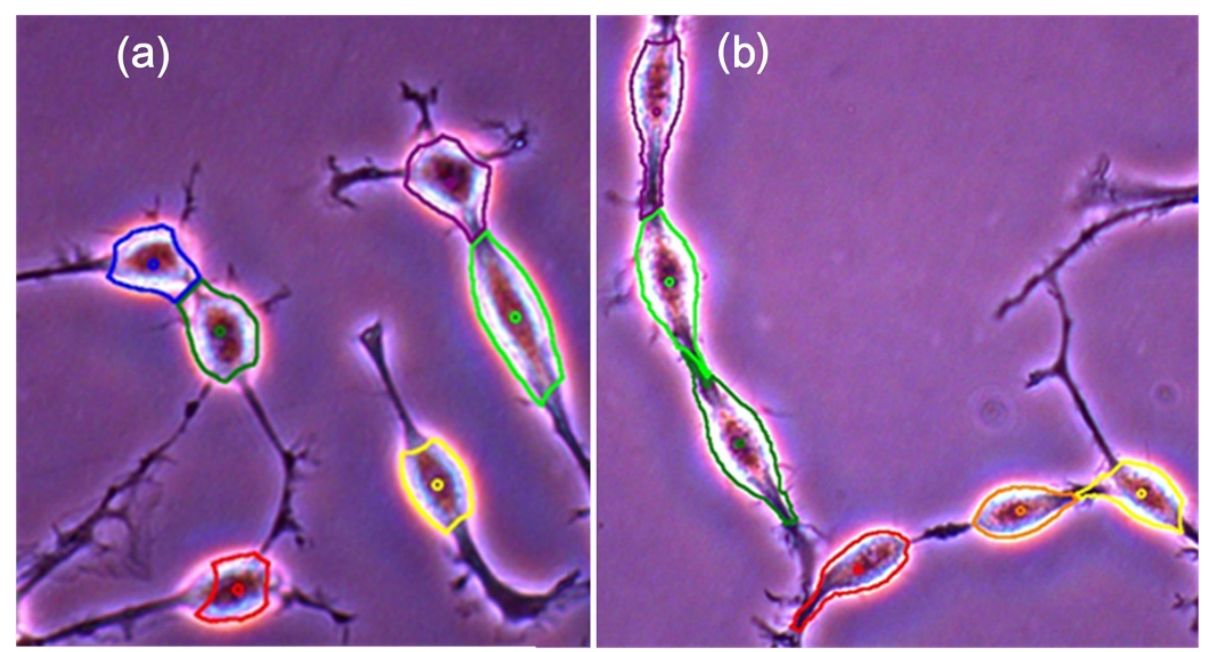

Figure 10 Segmentation of different groups of adjacent cells (a-b). The boundary of each cell is indicated by the line in different colors

for the identification. Firstly, the pipette should only be moved along the $\mathrm{x}$-direction. Secondly, the length of the pipette on the $x$-direction in the image should be longer than one-third of the image. The grayscale image of the pipette and the cells is shown in Figure 8a. As similar to the method for identification of cells, the dilation followed by erosion was used. The threshold operation was then used to obtain the binary image. After the binary image was obtained as shown in Figure 8c, all blobs of white pixels that were not connected to the left and right edge of the image were filled with black pixels as shown in Figure 8d. After this operation, the cells which are connected to the edge still appeared in the image. Afterwards, the blobs were filled by black pixels except the block that has the longest distance in the $\mathrm{x}$-direction as shown in Figure 8e. As from the prior calibration, the length of the pipette is longer than the length of the cell. The situation of some neighboring cells was also avoided by the operation of distance transform. The boundary of the pipette was identified, and the position of the tip of the pipette was determined.

\section{Results and discussion}

\section{Identification of different shapes of cells}

To further verify the identification method, cells in different shapes and sizes were tested and after obtaining the image of the cells, the detection and identification methods were applied to the images accordingly. As shown in Figure 9, different numbers and shapes of the cells were successfully identified, where their corresponding center and boundary were located and marked.

The identification and segmentation of the neighboring cells were also tested. The combination of the distance transform and the marker-based watershed transformation were applied to the images. The adjacent cells that were close to each other were successfully segmented, and the resulting images are shown in Figure 10.

\section{Yield of cell identification}

As the structural element and threshold value are the key parameters for the successful identification of cells, a batch of cells was tested independently, and a certain range of size of structural element and threshold values were applied. Experiments were conducted on 95 cells with the applied threshold value ranging from 130 to 250. The experiment was repeated for the changing applied size of structural element ranging from 31 to 61 . A cell was counted as successfully identified when its center and boundary were accurately located from the output image. The yield of cell identification is shown in

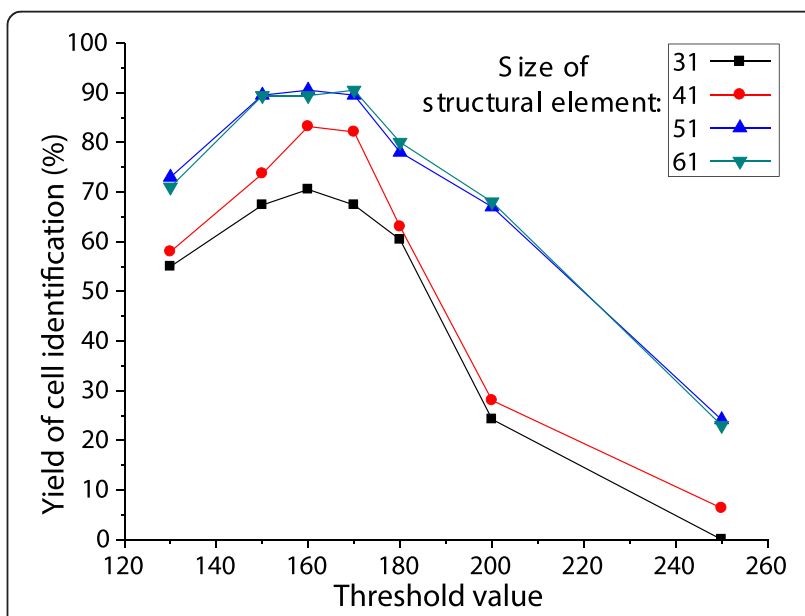

Figure 11 The yield of identification of cells on different sizes of structural element and threshold values. 


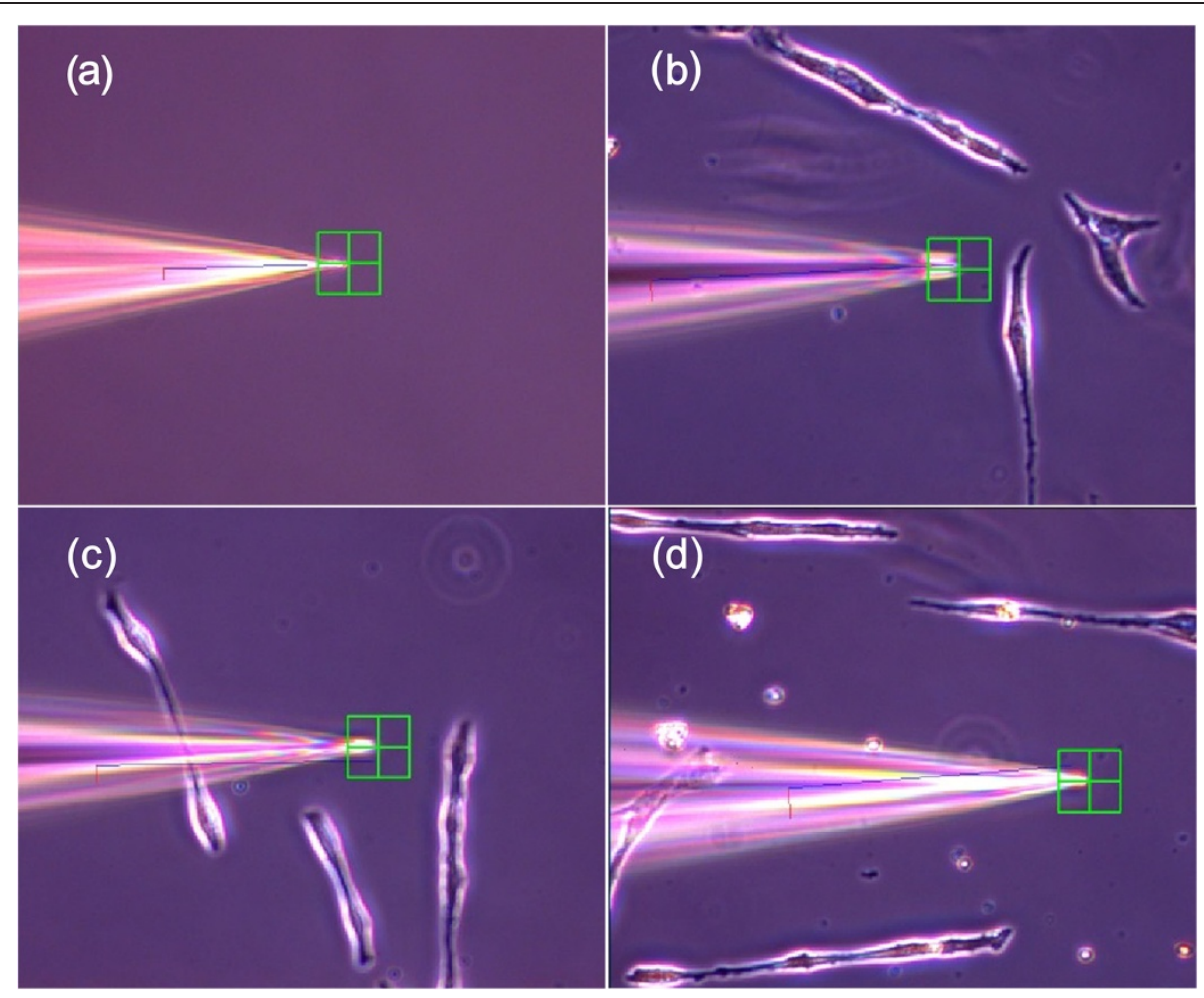

Figure 12 The images showing the successful identification of the pipette under different situations. The detected pipette was marked by the green box. (a) Identification of pipette without any cells. (b) Identification of pipette when couple cells are located around the pipette. (c) Identification of the pipette when couple cells are overlapped with the pipette. (d) Identification of the pipette when a batch of cells connected to each other.

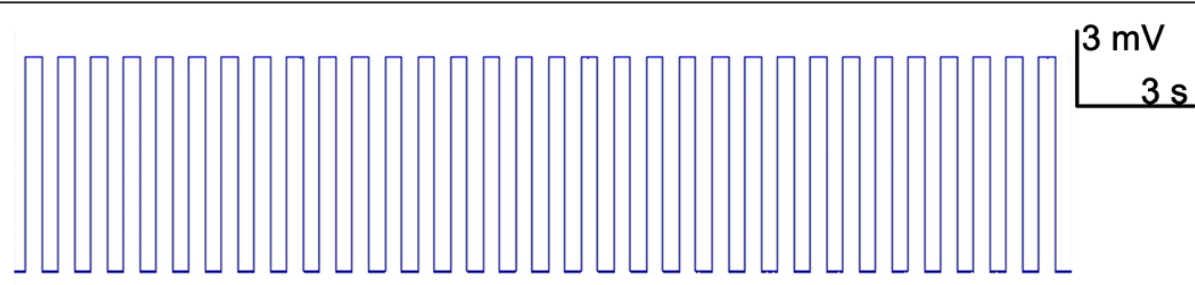

(a)

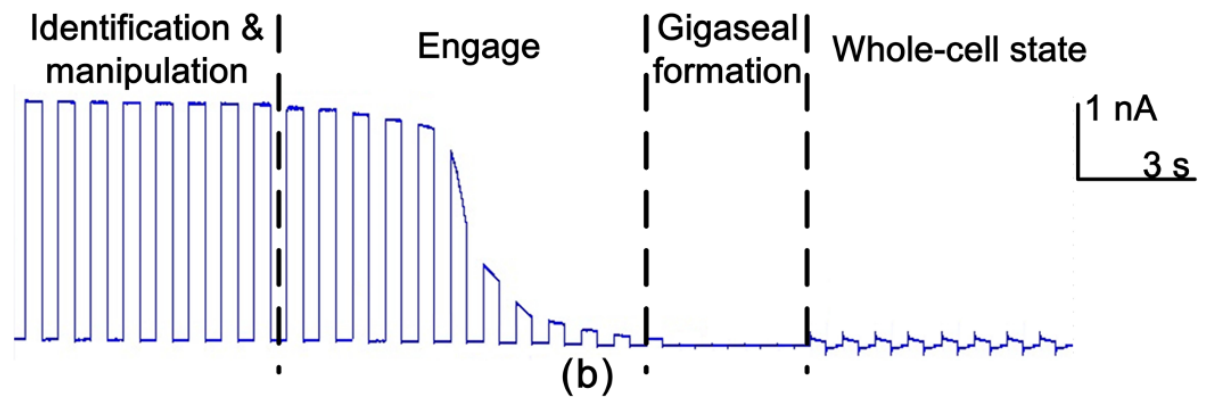

Figure 13 Patch clamp recording: (a) applied voltage pulse and (b) the induced transmembrane current. 
Table 1 Comparison of the gigaseal formation in manual and automated patch clamp process

\begin{tabular}{llc}
\hline & $\begin{array}{l}\text { Conditions of identification } \\
\text { of the cells }\end{array}$ & $\begin{array}{l}\text { Yield of gigaseal } \\
\text { formation (\%) }\end{array}$ \\
\hline $\begin{array}{l}\text { Manual } \\
\text { process }\end{array}$ & $\begin{array}{l}\text { One cell in the image can be } \\
\text { identified at a time }\end{array}$ & 69 \\
$\begin{array}{l}\text { Automated } \\
\text { process }\end{array}$ & $\begin{array}{l}\text { Ninety percent of the cells in the } \\
\text { image can be identified at a time }\end{array}$ & 72.4 \\
\hline
\end{tabular}

Figure 11. The results indicated that when the threshold value was 160 to 180 and the size of structural element was higher than 51, the highest yield of identification which was equal to $90 \%$ was obtained.

\section{Identification of pipettes}

Experiments were conducted for testing the identification of pipettes under different test conditions. As seen from Figure 12a, the individual pipette was successfully identified. When the cells were near the pipette, the system was also able to detect the pipette as shown in Figure 12b. As shown in Figure 12c, a more complex situation was tested in which the pipette was overlapped by the cells. When a batch of cells was connected and located near the pipette, the pipette was also successfully identified as shown in Figure 12d.

\section{Validation}

Based on the experimental results showing that the cells and pipettes were successfully identified by using the developed approach, the method was integrated to a micromanipulation system. After detecting and locating the cells and pipette, the manipulator of the system was controlled to move the tip of the pipette to the center of the cell for patch clamping. An automated patch clamp program that was previously developed by our group was used [28], and the whole-cell patch clamp recording was then performed. A voltage pulse with the peak voltage of $10 \mathrm{mV}$ (Figure 13a) was applied and the corresponding transmembrane current was monitored (Figure 13b), and thus the state of patch clamp was indicated. The results indicated that different values of transmembrane current were obtained at different state of the patch clamp process, from identification, engaging, and gigaseal formation to the whole-cell state.

The yield of gigaseal formation in manual process and automated process were analyzed and compared. In the manual process, only one cell was chosen at a time. By using the visual-based approach, around $90 \%$ of cells in a digital image were identified at a time. The yield of the gigaseal formation was studied in both manual and automated patch clamp process (29 selected cells for each process). In the manual process, $69 \%$ of cells achieved

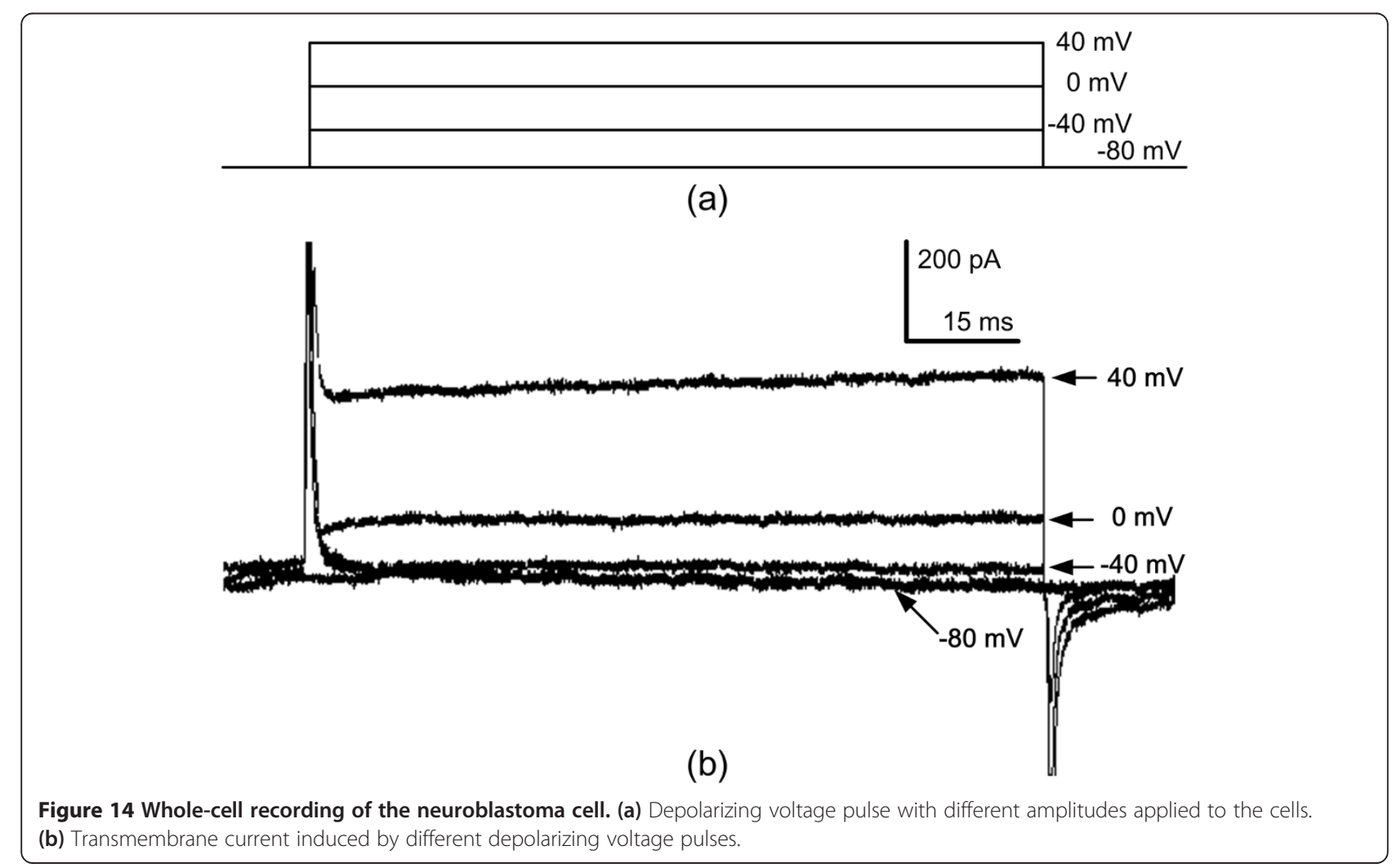


the gigaseal formation, and in the automated process, $72.4 \%$ of cells the achieved gigaseal formation. Therefore, the seal condition of the cell by using the automated patch clamp was comparable with seal condition which was obtained by using the manual process (Table 1).

The whole-cell recording was further performed by changing the voltage pulse amplitudes. The cell consists of various voltage-gated ion channels on cell membrane and the transmembrane currents, which indicated the ion channel response, were obtained and compared at different voltage pulse amplitudes. By applying the depolarizing voltage pulse to the cell, the voltage-gated ion channels can be activated and transmembrane current can be recorded. The transmembrane currents were induced using $100 \mathrm{~ms}$ depolarizing voltage pulse $(-40,0$, $40 \mathrm{mV}$ while holding potential $=-80 \mathrm{mV}$ ) as shown in Figure 14. A 20 to $30 \mathrm{pA}$ increment of transmembrane currents was obtained between -40 and $-80 \mathrm{mV}$, which showed that the voltage-gated ion channels were not activated at $-40 \mathrm{mV}$. The transmembrane current increased significantly when the voltage pulse was changed to 0 and $40 \mathrm{mV}$, which showed that the voltage-gated ion channels were activated by these voltage pulses. The curve of transmembrane currents is consistent with the related studies reported in [29], and this further validates the successful patch clamp recording using the method.

\section{Conclusions}

The identification method based on blob detection for the detection of cells and pipette was developed and applied for the automated patch clamp. The capability of identifying different shapes of adherent cells and pipettes was demonstrated. By combining the blob detection to distance transform and watershed algorithm, the automatic cell segmentation was realized. The automated patch clamp was implemented with the identification approach successfully. The method presented here provides a visual-based approach to the current automated patch clamp system that further facilitates the patch clamp recording.

\section{Competing interests}

The authors declare that they have no competing interests.

\section{Authors' contributions \\ RY developed the system, carried out the patch clamp experiments, and wrote the manuscript. RY, CHT, KLC, and KCW designed and implemented the visual based method. NX and JY participated in the design of the study. KWCL designed the study, developed the methodology, analyzed the data, and wrote the manuscript. All authors read and approved the final manuscript.}

\section{Acknowledgement}

The project described is supported in part by GRF grant from the Research Grant Council of the Hong Kong Special Administrative Region Government (CityU139313 and CityU124213).

\section{Author details}

'Department of Mechanical and Biomedical Engineering, City University of Hong Kong, Tat Chee Ave, Kowloon, Hong Kong. ${ }^{2}$ University of Science and Technology of China, Jinzhai RdShushan, Hefei, China. ${ }^{3}$ Michigan State University, Trowbridge Rd, East Lansing, Ml 48824, USA.

Received: 15 October 2014 Accepted: 5 November 2014

Published online: 05 December 2014

\section{References}

1. Jurkat-Rott K, Lehmann-Horn F (2004) The patch clamp technique in ion channel research. Curr Pharm Biotechnol 5(4):387-395

2. Ward JM, Mäser P, Schroeder JI (2009) Plant ion channels: gene families, physiology, and functional genomics analyses. Annu Rev Physiol 71:59-82

3. Kornreich BG (2007) The patch clamp technique: Principles and technical considerations. J Vet Cardiol 9(1):25-37

4. Kaczorowski GJ, McManus OB, Priest BT, Garcia ML (2008) Ion channels as drug targets: the next GPCRs. J Gen Physiol 131(5):399-405

5. Cang J, Isaacson J (2003) In vivo whole-cell recording of odor-evoked synaptic transmission in the rat olfactory bulb. J Neurosci 23:4108-4117

6. Kass R (2005) The channelopathies: novel insights into molecular and genetic mechanisms of human disease. J Clin Investig 115(8):1986-1989

7. Ahmed I, Bose SK, Pavese N, Ramlackhansingh A, Turkheimer F, Hotton G, Hammers A, Brooks DJ (2011) Glutamate NMDA receptor dysregulation in Parkinson's disease with dyskinesias. Brain 134(Pt 4):979-986

8. Zhao Y, Inayat S, Dikin D (2008) Patch clamp technique: review of the current state of the art and potential contributions from nanoengineering. J Nanoeng Nanosyst 222:1-11

9. Kodandaramaiah SB, Franzesi GT, Chow BY, Boyden ES, Forest CR (2012) Automated whole-cell patch-clamp electrophysiology of neurons in vivo. Nat Methods 9(6):1-6

10. Brueggemann A, George M, Klau M, Beckler M, Steindl J, Behrends JC, Fertig N (2004) lon channel drug discovery and research: the automated Nano-Patch-Clamp technology. Curr Drug Discov Technol 1(1):91-96

11. Fertig N, Blick R, Behrends J (2002) Whole cell patch clamp recording performed on a planar glass chip. Biophys J 82(6):3056-3062

12. Tang KC, Reboud J, Kwok YL, Peng SL, Yobas L (2010) Lateral patchclamping in a standard 1536-well microplate format. Lab Chip 10(8):1044-1050

13. Vasilyev D, Merrill T, Iwanow A, Dunlop J, Bowlby M (2006) A novel method for patch-clamp automation. Pflugers Arch - Eur J Physiol 452(2):240-247

14. Fejtl M, Czubayko U, Hümmer A, Krauter T, Lepple-Wienhues A (2007) Automated glass pipette-based patch-clamp techniques. Neuromethods 38:435-450

15. Vukicevic M, Weder G, Boillat A, Boesch A, Kellenberger S (2006) Trypsin cleaves acid-sensing ion channel 1a in a domain that is critical for channel gating. J Biol Chem 281(2):714-722

16. Wai Wong C, Dye DE, Coombe DR (2012) The role of immunoglobulin superfamily cell adhesion molecules in cancer metastasis. Int J Cell Biol 2012:340296

17. Ouyang WZPR, Zhang WJ, Gupta MM (2007) Overview of the development of a visual based automated bio-micromanipulation. Int Conf Comput Vision 17(10):147-151

18. Priyankara GPM, Seneviratne OW, Silva RKOH, Soysa WVD, De Silva CR (2011) An extensible computer vision application for blood cell recognition and analysis. IEEE Conf Comput Vision Pattern Recognit 805-902

19. Dewan MAA, Ahmad MO, Swamy MNS (2011) Tracking biological cells in time-lapse microscopy: an adaptive technique combining motion and topological features. IEEE Trans Biomed Eng 58(6):1637-1647

20. Damerval C, Meignen S (2007) Blob detection with wavelet maxima lines. IEEE Signal Processing Letters 14(1):39-42

21. Moon WK, Shen Y-W, Bae MS, Huang C-S, Chen J-H, Chang R-F (2013) Computer-aided tumor detection based on multi-scale blob detection algorithm in automated breast ultrasound images. IEEE Trans Med Imaging 32(7):1191-1200

22. Peng H, Zhou X, Li F, Xia X, Wong STC (2009) Integrating multi-scale blob/curvilinear detector techniques and multilevel sets for automated segmentation of stem cell images. In: Proceedings - 2009 IEEE International Symposium on Biomedical Imaging: From Nano to Macro, ISBI 2009., pp 1362-1365

23. Ouyang PR, Zhang WJ, Gupta MM, Zhao W (2007) Overview of the development of a visual based automated bio-micromanipulation system. Mechatronics 17(10):578-588 
24. Becker BC, Yang S, MacLachlan RA, Riviere CN (2012) Towards vision-based control of a handheld micromanipulator for retinal cannulation in an eyeball phantom. In: Proceedings of the IEEE RAS and EMBS International Conference on Biomedical Robotics and Biomechatronics., pp 44-49

25. Mason S (2003) Computer vision and image processing. ISPRS J Photogramm Remote Sens 48(2):24-27

26. Holzer S, Hinterstoisser S, llic S, Navab N (2009) Distance transform templates for object detection and pose estimation. In: 2009 IEEE Computer Society Conference on Computer Vision and Pattern Recognition Workshops, CVPR Workshops 2009., pp 1177-1184

27. Couprie M, Bertrand G, Psi L, Esiee G (2003) Topological grayscale watershed transformation. SPIE Vision Geometry VI Proceedings 3168:136-146

28. Yang R, Lai KWC, Xi N, Yang J (2013) Development of automated patch clamp system for electrophysiology. IEEE Int Conf Robotics Biomim ROBIO 2013:2185-2190

29. Reeve HL, Vaughan PF, Peers C (1992) Glibenclamide inhibits a voltage-gated $\mathrm{K}+$ current in the human neuroblastoma cell line SH-SY5Y. Neuroscience Letter 135(1):37-40

doi:10.1186/s40638-014-0020-5

Cite this article as: Yang et al:: Cell segmentation and pipette

identification for automated patch clamp recording. Robotics and

Biomimetics 2014 1:20.

\section{Submit your manuscript to a SpringerOpen ${ }^{\circ}$ journal and benefit from:}

- Convenient online submission

- Rigorous peer review

- Immediate publication on acceptance

- Open access: articles freely available online

- High visibility within the field

- Retaining the copyright to your article

Submit your next manuscript at $>$ springeropen.com 